\title{
Static strain and stress changes in eastern Japan due to the 2011 off the Pacific coast of Tohoku Earthquake, as derived from GPS data
}

\author{
Hiroaki Takahashi \\ Institute of Seismology and Volcanology, Hokkaido University, Sapporo 060-0810, Japan \\ (Received April 7, 2011; Revised June 14, 2011; Accepted June 25, 2011; Online published September 27, 2011)
}

\begin{abstract}
The 2011 M 9.0 Tohoku earthquake induced regional crustal deformation not only in the Japanese Islands but also in north-eastern Asia. Strain release due to mainshock faulting should cause strain redistribution in the overriding plates. The dense GPS network in Japan enables us to calculate co-seismic strain and stress changes from observed data. Strain is a more objective indicator than displacement because no reference frame is required. The co-seismic strain field clearly indicates island-scale strain redistribution. Huge extensional strain changes were concentrated in the southern Iwate and northern Miyagi regions, with a maximum value of $45 \times 10^{-6}$, which might correspond to approximately 225 to 450 years of strain accumulation. This implies relatively large strain accumulation and release in these regions. Small strain decay was observed in the northernmost NiigataKobe tectonic zone and a possible anomalous Coulomb failure stress change was observed in the Mt. Fuji region. Earthquakes triggered in the above regions might be associated with these anomalies, and/or these non-uniform crustal deformations may reflect crustal heterogeneity.
\end{abstract}

Key words: The 2011 off the Pacific coast of Tohoku Earthquake, GPS, principal strain, maximum shear strain, triggering earthquake, Coulomb failure stress change, subsurface structure.

\section{Introduction}

The great $M 9.02011$ off the Pacific coast of Tohoku Earthquake between subducting Pacific and overriding plates off north-eastern Japan (Fig. 1) generated widespread crustal deformation not only in the Japanese islands but also in north-eastern Asia. The co-seismic displacement field was observed in quasi-real time by the Japanese nationwide GPS network (GEONET) operated by the Geospatial Information Authority of Japan (GSI). Several meters of horizontal displacement near the epicenter were reported. Although GPS-derived crustal deformation is commonly expressed as displacement, this requires a reference station/frame because displacement is a relative value. Faulting is defined as the instantaneous release of accumulated strain on the fault plane. Therefore, expressing the strain as a displacement field is natural in evaluating the characteristics of faulting. This approach is also free from apparent shifts in the reference frame, which are present in displacement data.

Large earthquakes often induce remote seismicity and several actual examples have been reported (e.g., Protti et al., 1995; Brodsky et al., 2000; Ueda and Takahashi, 2005). Earthquakes possibly triggered by this $M 9.0$ mainshock have been observed in the region from north to central Japan. Static strain-stress change is a candidate for the driving force and so may become an indicator in the evaluation of triggering earthquake activity (e.g., Stein and

Copyright (c) The Society of Geomagnetism and Earth, Planetary and Space Sciences (SGEPSS); The Seismological Society of Japan; The Volcanological Society of Japan; The Geodetic Society of Japan; The Japanese Society for Planetary Sciences; TERRAPUB.

doi:10.5047/eps.2011.06.049
Lisowski, 1983; King et al., 1994). The static strain-stress change at each target point is usually calculated indirectly, through a model based on assumed mainshock fault parameters. This procedure is very convenient and can provide arbitrary density data. On the other hand, this method is strongly affected by the uncertainly of a priori fault parameters. The heterogeneity of the medium is also not considered. In other words, the strain-stress values obtained by the above method are only estimations, rather than actual observations.

The GEONET dense GPS network with a spacing of 20 to $30 \mathrm{~km}$ allows us to calculate strain tensor inner triangles from observed displacement data. The static stress change at each triangle can then be estimated from the strain tensor using proper elastic constants. In order to investigate the characteristics of the co-seismic strain-stress redistribution due to this great earthquake, we calculated strain-stress changes from GEONET GPS displacement data.

\section{Data and Method}

We first estimated the strain tensor and principal and maximum shear strains using GEONET F3 daily coordinate data, which is freely distributed by GSI. Co-seismic displacements were estimated from the daily coordinate differences of 10 and 12 for March 2011. Only horizontal components were derived. Triangulation of GEONET sites was performed by the TRIANGULATE command of GMT (Wessel and Smith, 1998). The strain tensor, principal strain and maximum shear strain within each triangle of the grid was computed using the displacements at each apex of a triangle (e.g., Terada and Miyabe, 1929; Tsuboi, 1933). The 


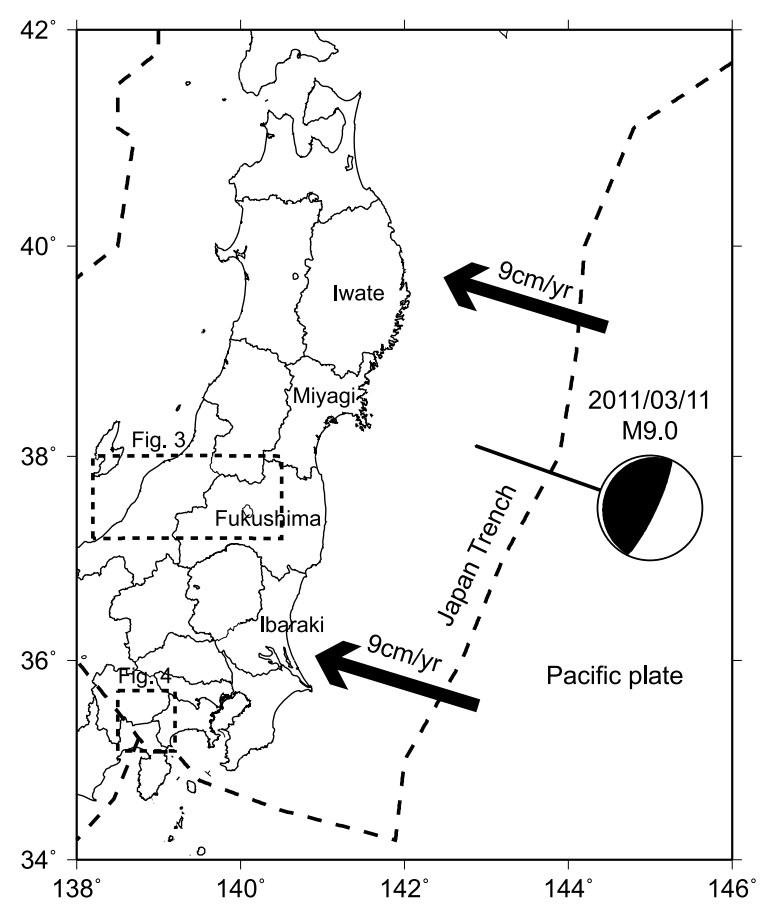

Fig. 1. Epicentral information of the mainshock and tectonic background. The epicenter location and mechanism information are obtained from the Japan Meteorological Agency and GCMT data, respectively. The Oceanic Pacific plate is subducting under the overriding continental plate at $9 \mathrm{~cm} /$ year (Sella et al., 2002).

maximum shear strain was calculated as follows:

$$
\left(e_{x y}^{2}+\left(e_{x x}-e_{y y}\right)^{2} / 4\right)^{0.5}
$$

The Coulomb failure stress change $(\Delta \mathrm{CFS})$ on the ground surface was estimated from the strain tensor with a Poisson ratio of 0.25 , a rigidity of $30 \mathrm{GPa}$ and an apparent friction coefficient of 0.4 .

\section{Results and Discussion}

\subsection{Spatial characteristics of strain change}

The co-seismic principal strain distribution is shown in Fig. 2(a). This map clearly indicates huge extensional principal strains toward the epicenter with values greater than $45 \times 10^{-6}$ observed in the southern Iwate and northern Miyagi regions. Eastward extension is observed in Fukushima and Ibaraki but the magnitude of the strain is less than that in southern Iwate and northern Miyagi. The orientation of the strain in this region is likely not due to dislocation of the epicenter. This might imply the existence of another asperity patch with a smaller slip off Fukushima and Ibaraki. These features agree well with slip distribution models estimated by seismic waves and GPS data (e.g., JMA web page, http://www.jma.go.jp, GSI web page, http://www.gsi.go.jp).

The distribution of maximum shear strain has clear spatial characteristics (Fig. 2(b)). Triangles with strains of more than $30 \times 10^{-6}$ were concentrated along the coastlines of southern Iwate and northern Miyagi. Concentric strain decay was observed at these locations. No triangles with anomalously large strains were observed in Fukushima or Ibaraki. These features might indicate that dominant mo-

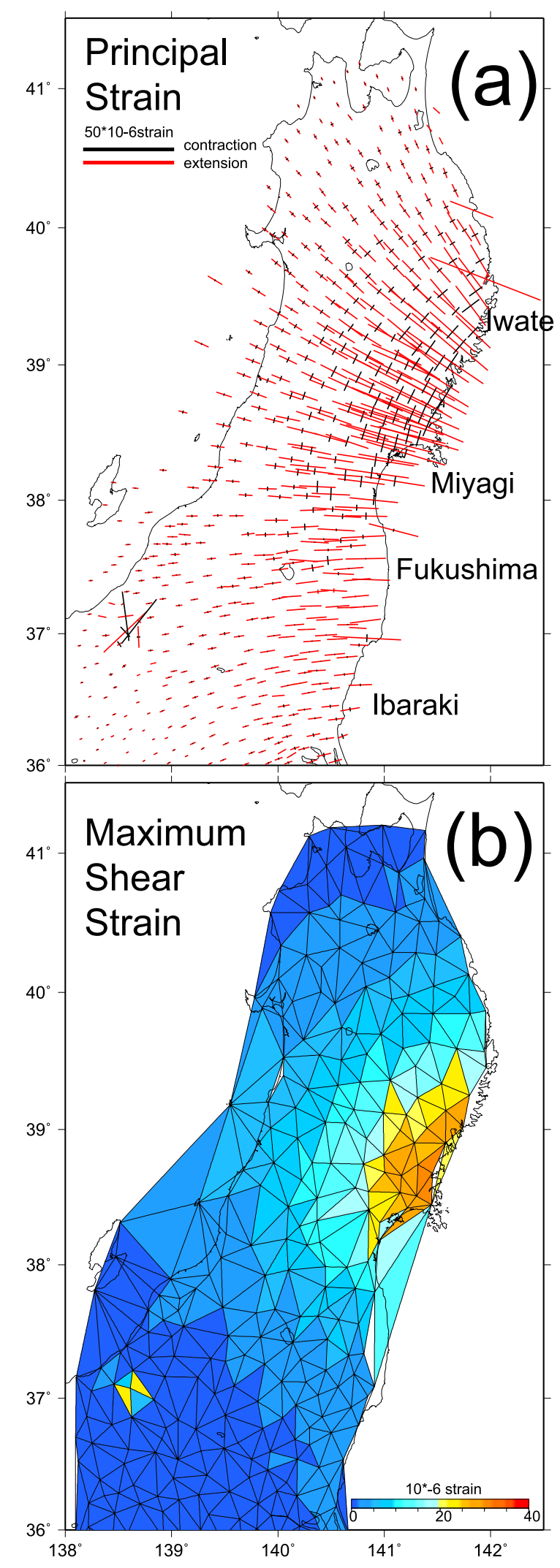

Fig. 2. Co-seismic strain changes in eastern Japan. (a) Principal strain, (b) maximum shear strain distributions.

ment release was limited off southern Iwate and northern Miyagi.

The compressional strain rate during recent decades along the Miyagi coastline region prior to the mainshock 


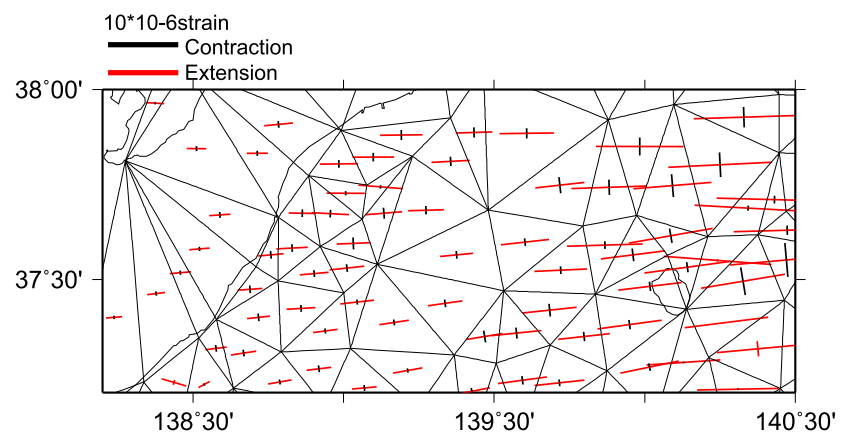

Fig. 3. Co-seismic principal strain distribution in the rectangle shown in Fig. 1. The western part of this region corresponds to the northernmost Niigata-Kobe tectonic zone, in which a high strain accumulation rate has been observed.

was approximately $0.1 \times 10^{-6}$ to $0.2 \times 10^{-6}$ per year (Kato et al., 1998; Sagiya et al., 2000; Miura et al., 2002). If we assume that this rate was constant during the entire interseismic period, the largest values of released strain $\left(45 \times 10^{-6}\right)$ correspond to approximately 225 to 450 years of strain accumulation. The occurrence of several M 7-class earthquakes (the 1897, 1936 and 1978 earthquakes of magnitude 7.4) might allow an even longer strain accumulation period. The absence of $M 8$-class events during the past several hundred years on the plate interface, however, implies that the impact of interseismic events on strain accumulation was less significant. Although the estimated earthquake magnitudes were smaller than that of the latest event, candidates for previous massive events were the 1611 Keicho $(M$ 8.1) and the 869 Jogan ( $M$ 8.3) earthquakes (Usami, 2003). The recurrence interval between these three events (742 and 400 years) appears to agree somewhat with the accumulation period estimated from strain data. Tsunami deposit data, however, implies longer periods between giant tsunamis (Minoura et al., 2001). In order to evaluate this hypothesis, more detailed investigations of previous great earthquakes are required.

Note that the anomaly at the south-western edge in Fig. 2 is the co-seismic effect of a $M 6.7$ earthquake that occurred during the observation period.

\subsection{Small strain attenuation in the northernmost} Niigata-Kobe tectonic zone

Figure 3 indicates the transversal principal strain distribution in the rectangular area shown in Fig. 1. The strain spatial decay rates in the western and eastern sides of E139.5 appear to be different. Although a homogeneous elastic medium requires strain decay as a function of distance, the attenuation rate at the western side is smaller than that at the eastern side. In addition, a $M 6.7$ crustal earthquake occurred eight hours after the mainshock in the south-western extension portion of this region. A co-seismic strain change due to this event appears in the south-western edge of Fig. 2. This anomalous region corresponds to the northernmost part of the Niigata-Kobe tectonic zone (e.g., Sagiya, 2000). A high strain accumulation rate has been observed by GPS and medium magnitude crustal earthquakes have occurred frequently in this region (e.g., $M 6.8$ in 2004 and $M 6.8$ in 2007). Seismic tomography investigations indicated a

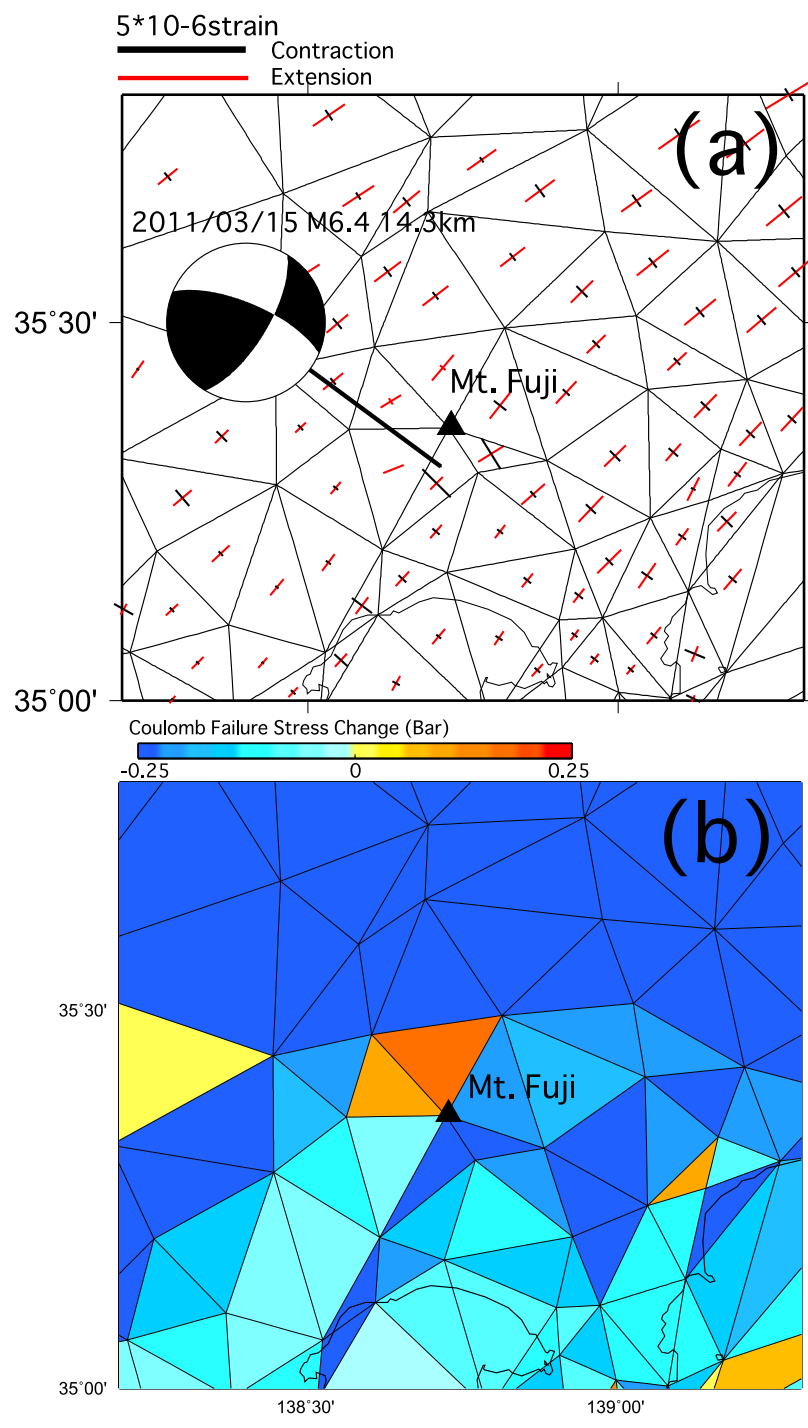

Fig. 4. (a) Co-seismic principal strain change around the active Mt. Fuji volcano region shown in Fig. 1. The epicenter and focal mechanism data are from the Japan Meteorological Agency and the F-net WWW server, respectively. (b) Coulomb failure stress changes ( $\triangle \mathrm{CFS}$ ) of each triangle obtained by assuming a NNE directional pure left-lateral fault plane of the mechanism solution.

clear low-velocity zone beneath this region (e.g., Nakajima and Hasegawa, 2007, 2008). The observed anomalous coseismic strain might reflect this heterogeneous structure. The characteristics of instantaneous co-seismic response and its difference with long-term strain accumulation might provide a clue to clarify the mechanism of strain concentration.

\subsection{Strain and stress anomalies in the Mt. Fuji area}

A $M 6.4$ crustal earthquake occurred just beneath the active Mt. Fuji volcanic mountain on 15 March 2011, four days after the mainshock. This earthquake might be the largest earthquake in the Mt. Fuji region during historical times.

We investigated the co-seismic strain and $\triangle \mathrm{CFS}$ changes due to the $M 9.0$ mainshock around this volcano. GPS data for analysis was obtained before this event. Principal strains of approximately $1 \times 10^{-6}$ were generally observed. Note that the direction and ratio of the maximum axis to the min- 
imum axis of principal strains at triangles around Mt. Fuji were slightly different from those of the surrounding area (Fig. 4(a)). Triangles showing anomalous strain were constructed with the Mt. Fuji GPS station situated at the top of mountain. Estimation of $\triangle \mathrm{CFS}$ by assuming NNE pure leftlateral strike slip, which might be a fault plane of the $M 6.4$ earthquake, is shown in Fig. 4(b). Enhancement of faulting in the northern Mt. Fuji region is indicated. The occurrence of the $M 6.4$ earthquake may support the reliability of the observed data. These observations may indicate a possible anomalous strain-stress change due to a heterogeneous subsurface structure (Nakamichi et al., 2007) and its relation to the triggering of earthquake activity in this region.

However, identification of this strain-stress anomaly is strongly dependent on the GPS station data at the summit of Mt. Fuji. In order to confirm the reliability of this result, investigation of data quality using a different data source should be performed in the future.

\section{Conclusion}

Co-seismic strain redistribution on the overriding plate due to the 2011 Tohoku earthquake was evaluated by observed GPS displacement data. Enormous strain changes exceeding $45 \times 10^{-6}$ were computed in the southern Iwate and northern Miyagi regions, but these values did not extend to the south. This might indicate a significant release of concentrated strain in these regions. A strain accumulation period of 225 to 450 years was estimated from the interseismic strain rate and the co-seismic strain magnitude. A small amount of strain attenuation was found in the northernmost Niigata-Kobe tectonic zone, which might reflect subsurface heterogeneity. Possible anomalous strain and stress changes were detected around the Mt. Fuji region, where a $M 6.4$ crustal earthquake occurred after the mainshock. This implies that this remote earthquake may have been a triggering event due to strain redistribution.

Acknowledgments. The authors would like to thank Drs. Kaj Johnson and Sigeru Nakao for reviewing the manuscript. We obtained GEONET GPS F3 coordinate data from the Geospatial Information Authority of Japan (http://www.gsi.go.jp). Focal mechanism data were obtained from the NIED F-net home page (http://www.fnet.bosai.go.jp) and the GCMT homepage (http://www.globalcmt.org). Hypocenter data were determined by JMA in cooperation with the Ministry of Education, Culture, Sports, Science and Technology (MEXT) in order to analyze seismic wave data provided by cooperating organizations, including Hokkaido Univ., Hirosaki Univ., Tohoku Univ., the Univ. of Tokyo, Nagoya Univ., Kyoto Univ., Kochi Univ., Kyushu Univ., Kagoshima Univ., the Nat. Res. Inst. Earth Sci. Disa. Prev., the Nat. Inst. Adv. Ind. Sci. Tech., the Tokyo Metropolitan Govern., Shizuoka Pref., the Hot Springs Res. Inst. Kanagawa Pref., Yokohama City, the GSI and the JAMSTEC. The present study was supported by the MEXT of Japan through the Observation and Research Program for Prediction of Earthquakes and Volcanic Eruptions and KAKENHI (21253005, 22253005).

\section{References}

Brodsky, E. E., V. Karakostas, and H. Kanamori, A new observation of dynamically triggered regional seismicity: Earthquake in Greece following the August, 1999 Izmit, Turkey Earthquake, Geophys. Res. Lett., 27, 2741-2744, 2000.

Kato, T., G. S. El-Fiky, and E. N. Oware, Crustal strains in Japanese islands as deduced from dense GPS array, Geophys. Res. Lett., 25, 3445-3448, 1998.

King, G. C. P., R. S. Stein, and J. Lin, Static stress changes and the triggering of earthquakes, Bull. Seismol. Soc. Am., 84, 935-953, 1994.

Minoura, K., F. Imamura, D. Sugawara, Y. Kono, and T. Iwashita, The 869 Jogan tsunami deposit and recurrence interval of large-scale tsunami on the Pacific coast of northeast Japan, J. Nat. Disaster Sci., 23, 83-88, 2001.

Miura, S., T. Sato, K. Tachibana, Y. Satake, and A. Hasegawa, Strain accumulation in and around Ou Backbone Range, northeastern Japan as observed by a dense GPS network, Earth Planets Space, 54, 10711076, 2002.

Nakajima, J. and A. Hasegawa, Deep crustal structure along the NiigataKobe Tectonic Zone, Japan: its origin and segmentation, Earth Planets Space, 59, e5-e8, 2007.

Nakajima, J. and A. Hasegawa, Existence of low-velocity zones under the source areas of the 2004 Chuetsu and 2007 Chuetsu-oki earthquakes inferred from travel-time tomography, Earth Planets Space, 60, 1127 1130, 2008.

Nakamichi, H., H. Watanabe, and T. Ohminato, Three-dimensional velocity Structure of Mount Fuji and the South Fossa magna, central Japan, J. Geophys. Res., 112, doi:10.1029/2005JB004161, 2007.

Protti, M., K. McNally, J. Pacheco, V. Gonzalez, C. Montero, J. Segura, J. Brenes, V. Barboza, E. Malavassi, F. Guendel, G. Simila, D. Rojas, A. Velasco, A. Mata, and W. Schillinger, The March 25, 1990 (MW=7.0, $\mathrm{ML}=6.8)$, earthquake at the entrance of the Nicoya Gulf, Costa Rica: Its prior activity, foreshocks, aftershocks, and triggered seismicity, $J$. Geophys. Res., 100, 20345-20358, 1995.

Sagiya, T., S. Miyazaki, and T. Tada, Continuous GPS array and presentday crustal deformation of Japan, Pure Appl. Geophys., 157, 2303 2322, 2000

Sella, G. F., T. H. Dixon, and A. Mao, REVEL: A model for recent plate velocities from space geodesy, J. Geophys. Res., 107, 2081, doi:10.1029/2000JB000033, 2002.

Stein, R. S. and M. Lisowski, The 1979 Homestean Valley Earthquake sequence, California: Control of aftershocks and postseismic deformation, J. Geophys. Res., 88, 6477-6490, 1983.

Terada, A. and N. Miyabe, Deformation of the earth crust in Kwansai district and its relation to the orographic features, Bull. Earthq. Res. Inst., Univ. Tokyo, 7, 233-239, 1929.

Tsuboi, C., Investigation on the deformation of the earth's crust found by precise geodetic means, Jpn. J. Astron. Geophys., 10, 93-248, 1933.

Ueda, M. and H. Takahashi, Triggering earthquakes along the volcanic front in the eastern Hokkaido, Japan, and its relevance to the static stress change due to the 2003 Tokachi-oki earthquake, J. Seismol. Soc. Jpn., $2, \mathbf{5 8}, 115-119,2005$.

Usami, T., Materials for Comprehensive List of Destructive Earthquakes in Japan (latest edition), University of Tokyo Press, p. 605, 2003.

Wessel, P. and W. H. F. Smith, New, improved version of the Generic Mapping Tools released, Eos Trans. AGU, 79, 579, 1998.

H. Takahashi (e-mail: hiroaki@mail.sci.hokudai.ac.jp) 